\title{
Endothelial function for the evaluation of anti-atherosclerotic drugs
}

\author{
Kenji Okumura and Xian Wu Cheng \\ Hypertension Research (2010) 33, 780-781; doi:10.1038/hr.2010.104; published online 17 June 2010
}

$\mathrm{C}^{\mathrm{n}}$ linical and experimental studies indicate that hypertension accelerates the development of arteriosclerosis. Atherosclerosis is a chronic inflammatory disease, and is characterized by the accumulation of a fibro-fatty plaque consisting of immune cells, vascular smooth muscle cells, vascular endothelial cells and the extracellular matrix, surrounding a lipid-rich core, leading to coronary heart disease and stroke. Recently, to detect early signs of atherosclerosis in vessels and predict the risk of future cardiovascular events, several imaging techniques, including vascular sonography, electron-beam and multi-slice computed tomography, and MRI, have been leveraged. As cardiovascular risk factors have been shown to cause endothelial dysfunction, ${ }^{1}$ endothelial function is considered to be impaired at an early phase of atherosclerosis.

Extensive epidemiologic evidence has consistently indicated that endothelial dysfunction has a pivotal role in the development of atherosclerosis and cardiovascular disease. ${ }^{2}$ Similarly, it is possible that assessment of endothelial function in the coronary or brachial arteries may predict long-term cardiovascular risk regardless of the method used for assessment. As nitric oxide (NO) has been found to be an endothelium-derived relaxant factor, ${ }^{3}$ it has become evident that the endothelium is an active paracrine, endocrine and autocrine organ that regulates vascular tone and structure and maintains vascular homeostasis. Moreover, the endothelium controls coagulation and inflammatory responses and, as a consequence, can prevent

$\overline{\text { Professor K Okumura and XW Cheng are at the Depart- }}$ ment of Cardiovascular Medicine, Nagoya University Graduate School of Medicine, Nagoya, Japan.

E-mail: kenji@med.nagoya-u.ac.jp atherosclerotic lesions from progressing into pathological inflammatory processes 4 (Table 1). One way of assessing endothelial dysfunction is by physiologically estimating it as an alteration of vasomotor activity. The disordered function of baseline activity and inadequate response of the endothelium to mechanical and pharmacological stimuli have been implicated as early markers in the development of atherosclerosis. ${ }^{1,2} \mathrm{NO}$ has an important role in the regulation of vascular tone, is produced by endothelial cells and causes the relaxation of vascular smooth muscle cells. Clearly, impairment of endothelium-dependent vasodilation represents endothelial dysfunction and is thought to be chiefly attributed to decreased endothelial NO bioavailability. Given the evidence supporting a pathogenic role of the endothelium, endothelial dysfunction is thought to be an early event in the atherosclerotic process that precedes formation of plaques before clinical detection of disease. ${ }^{5}$

Excess production of reactive oxygen species (ROS) leads to oxidative stress and decreases the bioavailability of NO. Under physiological conditions, ROS are balanced by a range of antioxidant defenses such as ascorbic acid (vitamin C), $\alpha$-tocophenol (vitamin E), and antioxidant enzymes such as the superoxide dismutases, catalase and the gluthathione peroxidases. Production of ROS in vascular tissue is markedly increased under the stressful conditions of hypercholesterolemia, diabetes mellitus and hypertension, implying one reason why NO bioavailability is reduced in such pathological conditions. Therefore, inactivation of $\mathrm{NO}$ can be induced not only by increased generation of ROS but also by a deficiency of ROS scavenging enzymes.

Using high-resolution ultrasound, Celermajer et al. ${ }^{6}$ introduced the measurement of flow-mediated dilation of the brachial artery as a non-invasive endothelial function test; they showed that non-invasive assessment is clinically beneficial to determine the presence of endothelial dysfunction in high-risk populations. This ultrasound method uses a stimulus of shear-mediated activation of endothelial nitric oxide synthase. Thereafter, this approach has been used and documented in the literature by numerous groups throughout the world as a tool for evaluating endothelial dysfunction in humans. However, standardization of its methodology must precede its clinical application. The reproducibility of flow-mediated dilation assessed by brachial artery ultrasound is not satisfactory in certain laboratories and can likely render inaccurate measurements for clinical research. ${ }^{7}$

Improved or recovered endothelial dysfunction by a therapeutic intervention such as pharmacotherapy, diet, exercise and apheresis is anticipated to be associated with beneficial outcomes and clinical prognosis. It is well known that statins can restore endothelial function in patients with hypercholesterolemia. Similarly, cholesterollowering treatments such as cholestyramine and LDL apheresis, angiotensin-converting enzyme inhibitors, ${ }^{8}$ antioxidants and antidiabetic drugs, including thiazolidinediones as peroxisome proliferator-activated receptor$\gamma$ agonists, are also reported to improve or recover endothelial dysfunction.

In this issue of the Hypertension Research, Wago et al. ${ }^{9}$ report long-term treatment with telmisartan, a selective antagonist for angiotensin type-1 receptor and a partial agonist for peroxisome proliferator-activated receptor- $\gamma$, in patients with both hypertension and type 2 diabetes mellitus. They showed that telmisartan had favorable effects on endothelial 
Table 1 Endothelial function

\section{Regulation of vascular tone}

Antioxidant effects

Anti-inflammatory effects

Antihypertrophic effects

Antithrombotic effects

Anticoagulant effects

Profibrinolytic effects

function and plasma adiponectin levels, in addition to lowering blood pressure and improving diabetes mellitus symptoms, dyslipidemia and plasma high-sensitivity C-reactive protein levels. Interestingly, they show that two factors, revealed by multivariate analysis, adiponectin levels and office systolic blood pressure (not home blood pressure), are independent contributors for improvement of endothelial function of the brachial artery. These findings indicate that elevated plasma adiponectin levels can act as a surrogate marker for treatment and prevention of the development of atherosclerosis accompanied by hypertension and diabetes mellitus.

We previously reported the close relationship between endothelial function and adiponectin levels, particularly the high-molecularweight form of adiponectin, which is the biologically active form of circulating adiponectin. ${ }^{10}$ Adiponectin is present abundantly in plasma and is decreased in conditions such as obesity, type 2 diabetes mellitus, coronary artery disease and hypertension, suggesting that dysregulated plasma adiponectin increases susceptibility to atherosclerotic vascular diseases. In addition to improving insulin sensitivity, adiponectin has been shown to have a direct effect on vessel walls. Plasma adiponectin accumulates rapidly in the subendothelial space of injured human arteries. As a result, adiponectin is able to enhance NO production in endothelial cells via AMP-activated protein kinase ${ }^{11}$ or phosphatidylinositol 3-kinase-dependent pathways. ${ }^{12}$ Hence, adiponectin might be an endogenous
Table 2 Effects of adiponectin

Glucose uptake in tissues

Increasing insulin sensitivity

Inhibition of gluconeogenesis in liver

Free-fatty acid oxidation in skeletal muscle and liver

NO production in endothelium

Anti-inflammatory effects

modulator of endothelial cell function. Another possible explanation of its relationship with endothelial function is that adiponectin may serve as an anti-inflammatory molecule for vascular walls that can attenuate the excessive inflammatory responses in vascular walls and prevent vascular remodeling (Table 2).

The adiponectin-elevating effects of medications have also been shown by treatment with the angiotensin II receptor blocker candesartan or the angiotensin-converting enzyme inhibitor temocapril, and have been associated with increasing insulin sensitivity with no change in adiposity. ${ }^{13}$ Another angiotensin II receptor blocker, valsartan, ${ }^{14}$ also increased adiponectin levels in obese subjects with normal glucose tolerance. These results indicate that each renin-angiotensin system blocker, even without an agonist for peroxisome proliferator-activated receptor- $\gamma$, appears to be able to increase adiponectin levels. In the future, it will be necessary to provide clinical evidence that the prevention of overt cardiovascular diseases can be achieved by increasing adiponectin levels induced by renin-angiotensin system blockers, such as telmisartan, regardless of lowering blood pressure.

1 Reddy KG, Nair RN, Sheehan HM, Hodgson JM. Evidence that selective endothelial dysfunction may occur in the absence of angiographic or ultrasound atherosclerosis in patients with risk factors for atherosclerosis. J Am Coll Cardiol 1994; 23: 833-843.
2 Suwaidi JA, Hamasaki S, Higano ST, Nishimura RA, Holmes Jr DR, Lerman A. Long-term follow-up of patients with mild coronary artery disease and endothelial dysfunction. Circulation 2000; 101: 948-954.

3 Furchgott RF, Zawadzki JV. The obligatory role of endothelial cells in the relaxation of arterial smooth muscle by acetylcholine. Nature 1908; 288: 373-376.

4 Vita JA, Keaney Jr JF, Larson MG, Keyes MJ, Massaro JM, Lipinska I, Lehman BT, Fan S, Osypiuk E, Wilson PW, Vasan RS, Mitchell GF, Benjamin EJ. Brachial artery vasodilator function and systemic inflammation in the Framingham Offspring Study. Circulation 2004; 110: 3604-3609.

5 Healy B. Endothelial cell dysfunction: an emerging endocrinopathy linked to coronary disease. J Am Coll Cardiol 1990; 16: 357-358.

6 Celermajer DS, Sorensen KE, Bull C, Robinson J, Deanfield JE. Endothelium-dependent dilation in the systemic arteries of asymptomatic subjects relates to coronary risk factors and their interaction. J Am Coll Cardiol 1994; 24: 1468-1474.

7 Hardie KL, Kinlay S, Hardy DB, Wlodarczyk J, Silberberg JS, Fletcher PJ. Reproducibility of brachial ultrasonography and flow-mediated dilatation (FMD) for assessing endothelial function. Aust N Z J Med 1997; 27: 649-652.

8 Morimoto S, Maki K, Aota Y, Sakuma T, Iwasaka T. Beneficial effects of combination therapy with angiotensin II receptor blocker and angiotensin-converting enzyme inhibitor on vascular endothelial function. Hypertens Res 2008; 31: 1603-1610.

9 Wago T, Yoshimoto T, Akaza I, Tsuchiya K, Izumiyama H, Doi M, Hirata Y. Improvement of endothelial function in patients with hypertension and type 2 diabetes after treatment with telmisartan. Hypertens Res 2010; 33: 796-801.

10 Torigoe M, Matsui H, Ogawa Y, Murakami H, Murakami R, Cheng XW, Numaguchi Y, Murohara T, Okumura K. Impact of the high-molecular-weight form of adiponectin on endothelial function in healthy young men. Clin Endocrinol (Oxf) 2007; 67: 276-281.

11 Ouchi N, Kobayashi H, Kihara S, Kumada M, Sato K, Inoue T, Funahashi T, Walsh K. Adiponectin stimulates angiogenesis by promoting cross-talk between AMP-activated protein kinase and Akt signaling in endothelial cells. J Biol Chem 2004; 279: 1304-1309.

12 Chen $\mathrm{H}$, Montagnani M, Funahashi T, Shimomura I, Quon MJ. Adiponectin stimulates production of nitric oxide in vascular endothelial cells. J Biol Chem 2003; 278: 45021-45026.

13 Furuhashi M, Ura N, Higashiura K, Murakami H, Tanaka M, Moniwa N, Yoshida D, Shimamoto K. Blockade of the renin-angiotensin system increases adiponectin concentrations in patients with essential hypertension. Hypertension 2003; 42: 76-81.

14 Pscherer S, Heemann U, Frank H. Effect of reninangiotensin system blockade on insulin resistance and inflammatory parameters in patients with impaired glucose tolerance. Diabetes Care 2010; 33: 914-919. 